\title{
The impact of conventional dietary intake data coding methods on foods typically consumed by low-income African-American and White urban populations
}

\author{
Marc A Mason ${ }^{1}$, Marie Fanelli Kuczmarski ${ }^{2, *}$, Deanne Allegro ${ }^{2}$, Alan B Zonderman ${ }^{1}$ and \\ Michele K Evans ${ }^{1}$ \\ 'Laboratory of Epidemiology and Population Sciences, National Institute on Aging, National Institutes of Health, \\ Baltimore, MD USA: ${ }^{2}$ Department of Behavioral Health and Nutrition, 010 Carpenter Sports Building, University of \\ Delaware, Newark, DE 19716, USA
}

Submitted 22 October 2013: Final revision received 12 0ctober 2014: Accepted 15 0ctober 2014: First published online 1 December 2014

\begin{abstract}
Objective: Analysing dietary data to capture how individuals typically consume foods is dependent on the coding variables used. Individual foods consumed simultaneously, like coffee with milk, are given codes to identify these combinations. Our literature review revealed a lack of discussion about using combination codes in analysis. The present study identified foods consumed at mealtimes and by race when combination codes were or were not utilized.

Design: Duplicate analysis methods were performed on separate data sets. The original data set consisted of all foods reported; each food was coded as if it was consumed individually. The revised data set was derived from the original data set by first isolating coded foods consumed as individual items from those foods consumed simultaneously and assigning a code to designate a combination. Foods assigned a combination code, like pancakes with syrup, were aggregated and associated with a food group, defined by the major food component (i.e. pancakes), and then appended to the isolated coded foods.

Setting: Healthy Aging in Neighborhoods of Diversity across the Life Span study. Subjects: African-American and White adults with two dietary recalls ( $n$ 2177).

Results: Differences existed in lists of foods most frequently consumed by mealtime and race when comparing results based on original and revised data sets. African Americans reported consumption of sausage/luncheon meat and poultry, while ready-to-eat cereals and cakes/doughnuts/pastries were reported by Whites on recalls.

Conclusions: Use of combination codes provided more accurate representation of how foods were consumed by populations. This information is beneficial when creating interventions and exploring diet-health relationships.
\end{abstract}

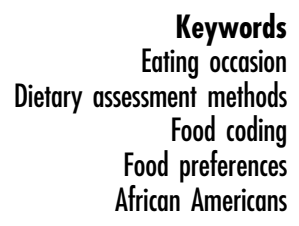

The results of dietary studies are influenced not only by the dietary collection method but also by the method of coding dietary data and of creating food groupings. There is no gold standard for the analysis of food intake data based on coded dietary recalls or records, especially items that are comprised of multiple food components. In the current paper these items are referred to as 'combinations'. A fast-food sandwich might be coded as a single item, while a self-prepared sandwich could be coded by each ingredient and a second code may be given indicating the item is comprised of several foods. Oftentimes in dietary pattern analyses, food mixtures are disaggregated into specific ingredients and each ingredient is then assigned to a disparate food group ${ }^{(1)}$. Depending on the use of coding variables in analysis, foods usually consumed by a population may be obscured or artificially separated, which can result in erroneous and misleading results ${ }^{(2)}$. For instance, if the investigator wanted to determine the quantity of milk consumed as a beverage and based the analysis using only the unique code for milk, then milk added to cereals would be included in this calculation ${ }^{(3)}$. To be accurate the investigator would need to use the codes for milk while excluding codes indicating when milk was part of a combination. Similar to the selection of a dietary method, the coding variables used in dietary data analysis must match the purpose of the study. 
The Dietary Intake Data System developed by the US Department of Agriculture (USDA) is used to collect dietary data for many projects, including What We Eat in America (WWEIA), the National Health and Nutrition Examination Survey (NHANES) and the Healthy Aging in Neighborhoods of Diversity across the Life Span (HANDLS) study. The main components of this system include three separate computer programs in succession: (i) the Automated Multiple-Pass Method (AMPM) for collecting $24 \mathrm{~h}$ recalls using standardized questions for all types of foods; (ii) the Post-Interview Processing System (PIPS) for extracting the data from the AMPM, arranging the data into formats suitable for loading into Survey Net and automating some of the food coding; and (iii) Survey Net for final coding, editing and nutritional analysis $^{(4)}$. The components of the USDA Food and Nutrient Database for Dietary Studies (FNDDS) are essential for collecting, processing and analysing food intake data using the USDA Dietary Intake Data System.

All foods collected in the AMPM are matched to food descriptions and their codes during processing in either PIPS or Survey Net. A coding pathway leads to a specific food code through the responses that have been provided for the questions asked about a food in the AMPM. AMPM also assigns combination codes. When intakes are coded using FNDDS in the Survey Net coding software, combination codes assigned initially by AMPM can be changed, removed or new ones added. A combination code identifies foods that were consumed simultaneously as one item. Individual foods in the combination are coded with their own separate food codes and amounts (Table 1). Combinations include one food added to another, such as sugar to coffee, and foods with separate ingredients, such as salads and sandwiches. This information allows researchers the ability to create a composite food and assign this item (such as the self-prepared sandwich) one code.

The public-use dietary data sets for WWEIA, NHANES and the HANDLS study contain coded foods. Variables in the dietary data sets for both of these projects include food codes, nutrients and combination food numbers and types $^{(5,6)}$. These data are used to explore the relationships between diet and health ${ }^{(7-10)}$. However, an inconsistency within coding exists. For example, a named brand of fastfood sandwich is given a single code while a sandwich made at home is coded by ingredient and then assigned a combination code. To implement a consistent approach to coding, researchers should either aggregate foods using the combination codes or disaggregate the composite foods prior to dietary analysis. When trying to create a picture of how foods are actually consumed, the implementation of the combination codes is essential. Yet our review of the literature revealed either a lack of detailed description as to the use of food combination codes ${ }^{(11)}$ or exclusion of food combinations in analysis ${ }^{(3,12)}$.

There is limited knowledge of foods as typically consumed at mealtimes by urban African-American and White populations $^{(13-16)}$. Perhaps the disparities in health of the poor that exist in the USA may be associated with not only the food choices, but also how these foods are prepared. The use of combination codes might reveal more insight into how these populations eat. With a better understanding of eating practices by these populations, nutrition educators, health professionals and public health policy makers may be able to translate nutrition goals into practical, culturally relevant and sex-specific diet recommendations ${ }^{(17,18)}$.

The main objective of the present study was to compare two coding methods to determine which method provided the best depiction of how foods were typically consumed at three mealtimes by the participants in the HANDLS study. Another aim was to determine any changes in the lists of foods consumed by race as reported on $24 \mathrm{~h}$ recalls for this low-income urban population resulting from the coding method.

\section{Methods}

\section{Background on the Healthy Aging in Neighborboods of Diversity across the Life Span study}

The HANDLS study, a community-based, prospective epidemiological study, was designed to examine whether race and socio-economic status influence age-related health disparities independently or synergistically. Participants

Table 1 Food and combination codes for selected foods

\begin{tabular}{|c|c|c|c|c|}
\hline Time eaten & Food description & Code & Grams eaten & Combination food type - food number* \\
\hline 7:00 am & Two egg omelette & 32105000 & 228 & \\
\hline 7:00 am & Coffee, regular brewed & 92101000 & 414 & $1 \dagger-1$ \\
\hline $7: 00$ am & Sugar & 91101010 & 14 & $1-1$ \\
\hline 7:00 am & English muffin, whole wheat & 51202000 & 58 & $3 \ddagger-2$ \\
\hline 7:00 am & Jelly, strawberry & 91401000 & 19 & $3-2$ \\
\hline $1: 00 \mathrm{pm}$ & Ice tea, pre-sweetened & 92301060 & 237 & \\
\hline $1: 00 \mathrm{pm}$ & Shrimp, steamed & 26319130 & 70 & $12 \S-3$ \\
\hline $1: 00 \mathrm{pm}$ & Cocktail sauce & 74406500 & 51 & $12-3$ \\
\hline 4:00 pm & Coffee, regular brewed & 92101000 & 414 & $1+-4$ \\
\hline $4: 00 \mathrm{pm}$ & Sugar & 91101010 & 14 & $1-4$ \\
\hline
\end{tabular}

*Food type indicates the food with additions or components of salads and sandwiches; food number indicates the items eaten simultaneously at a given time. $\dagger 1=$ beverages with additions.

$\ddagger 3=$ bread/baked products with additions

$\S 12=$ meat, poultry, fish with additions. 
were drawn from thirteen predetermined neighbourhoods in Baltimore City, yielding representative distributions of individuals between 30 and 64 years old who were African Americans and Whites, men and women, and of lower $(<125 \%$ of the US federal poverty guidelines (poverty income ratio)) and higher ( $>125 \%$ of the poverty income ratio) socio-economic status. The heuristic study design is a factorial cross of four factors: age, sex, race and socioeconomic status, with approximately equal numbers of individuals per 'cell'. There were two phases in the baseline HANDLS study. The first phase was done in the participant's home. This phase consisted of an in-home interview that included questionnaires about the participant's health status, health service utilization, psychosocial factors, dietary recall, neighbourhood characteristics and demographics. The second phase was completed 4 to $10 \mathrm{~d}$ later, on mobile research vehicles located in the preselected census tracts where participants resided. This part included a medical history and physical examination, dietary recall, cognitive evaluation, psychophysiology assessments including heart rate variability, arterial thickness, carotid ultrasound, assessments of muscle strength and bone density, and laboratory measurements. The study was conducted according to the guidelines laid down in the Declaration of Helsinki and all procedures were approved by the Institutional Review Boards at MedStar Health Research Institute and the University of Delaware. Written informed consent was obtained from all HANDLS participants who were compensated monetarily. Further detailed information on the study design, eligibility and recruitment of participants and the data collected can be found elsewhere ${ }^{(19,20)}$.

\section{Study sample}

Baseline data collection on socio-economically diverse African Americans and Whites began in August 2004 and ended in March 2009, with a total of 3720 participants. The sample consisted of 2177 individuals (59\% of respondents) who completed two days of $24 \mathrm{~h}$ dietary recalls (Fig. 1). Participants who completed only one recall day ( $n$ 1543) were not included because two days of recall provides a better representation of usual intakes. There were no statistical differences in demographic data or energy and nutrient profiles of the participants who completed one or both days of dietary recall. Thus the study sample is considered representative of the entire HANDLS baseline sample.

Characteristics of the HANDLS study participants are provided in Table 2 . The mean age of the sample was 47.8 (SE $0 \cdot 2)$ years and approximately half $(57 \%)$ was female. The racial composition was 58\% African American and $42 \%$ White. Self-reported socio-economic status revealed $43 \%$ with a household income $<125 \%$ and $57 \%$ with a household income $\geq 125 \%$ of the 2003 US Department of Health and Human Services poverty guidelines ${ }^{(21)}$. About 
Table 2 Characteristics of the participants by race and gender ( $n$ 2177), Healthy Aging in Neighborhoods of Diversity across the Life Span (HANDLS) study

\begin{tabular}{|c|c|c|c|c|c|c|c|c|}
\hline \multirow[b]{3}{*}{ Characteristic } & \multicolumn{4}{|c|}{ African Americans ( $n$ 1261) } & \multicolumn{4}{|c|}{ Whites $(n$ 916) } \\
\hline & \multicolumn{2}{|c|}{ Females ( $n$ 708) } & \multicolumn{2}{|c|}{ Males $(n 553)$} & \multicolumn{2}{|c|}{ Females ( $n$ 524) } & \multicolumn{2}{|c|}{ Males $(n$ 392) } \\
\hline & Mean or \% & $\mathrm{SE}$ & Mean or \% & SE & Mean or \% & SE & Mean or $\%$ & SE \\
\hline Age (years)* & 47.9 & 0.3 & $47 \cdot 7$ & 0.4 & $47 \cdot 7$ & 0.4 & $48 \cdot 1$ & 0.5 \\
\hline Education (\% <HS/GED) & 31.5 & $1 \cdot 7$ & $35 \cdot 8$ & $2 \cdot 0$ & 30.9 & $2 \cdot 0$ & $32 \cdot 1$ & 2.4 \\
\hline WRAT literacy ( $\% \leq 8$ th grade) & 47.4 & 1.9 & 49.5 & $2 \cdot 1$ & $25 \cdot 7$ & 1.9 & $26 \cdot 9$ & $2 \cdot 2$ \\
\hline Poverty status ( $\%<125 \%$ DHHS 2003 PIR) & 53.1 & 1.9 & $48 \cdot 3$ & $2 \cdot 1$ & $35 \cdot 7$ & $2 \cdot 1$ & $26 \cdot 5$ & $2 \cdot 2$ \\
\hline Employed in last month (\% unemployed) & 48.9 & 1.9 & 43.0 & $2 \cdot 1$ & $45 \cdot 0$ & $2 \cdot 2$ & $30 \cdot 9$ & $2 \cdot 3$ \\
\hline Self-reported health status (\% fair/poor) & $25 \cdot 7$ & 1.6 & $24 \cdot 8$ & 1.8 & $26 \cdot 9$ & 1.9 & $24 \cdot 2$ & $2 \cdot 2$ \\
\hline Hypertension (\%) & $53 \cdot 1$ & 1.9 & $44 \cdot 6$ & $2 \cdot 1$ & $35 \cdot 8$ & $2 \cdot 1$ & 43.9 & 2.5 \\
\hline Smoking (\% currently) & 43.9 & 1.9 & $58 \cdot 8$ & $2 \cdot 1$ & $44 \cdot 2$ & $2 \cdot 2$ & $46 \cdot 5$ & 2.5 \\
\hline $\mathrm{BMI}\left(\mathrm{kg} / \mathrm{m}^{2}\right)^{*}$ & 31.5 & 0.3 & 27.4 & 0.3 & $30 \cdot 8$ & 0.4 & 28.9 & 0.3 \\
\hline Obesity: $\mathrm{BMI} \geq 30 \mathrm{~kg} / \mathrm{m}^{2}$ (\%) & $53 \cdot 1$ & 1.9 & 27.9 & 1.9 & $45 \cdot 6$ & $2 \cdot 2$ & $36 \cdot 7$ & $2 \cdot 4$ \\
\hline Usual energy intake $(\mathrm{kJ})^{\star}$ & 7518 & 519 & 10217 & 544 & 7644 & 92 & 10724 & 163 \\
\hline Usual energy intake (kcal)* & 1812 & 124 & 2442 & 130 & 1827 & 22 & 2564 & 39 \\
\hline
\end{tabular}

HS, high school; GED, General Educational Development; WRAT, Wide Range Achievement Test; DHHS, US Department of Health and Human Services; PIR, poverty income ratio.

${ }^{*}$ Values are presented as means with their standard errors.

one-third of the sample had less than a high-school education. Among African Americans, $48 \%$ had less than an 8th grade literacy rate compared with $26 \%$ among Whites. Approximately one in four participants rated their health as fair/poor. Over $40 \%$ were current smokers. While the mean usual energy intake of the women was roughly $7531 \mathrm{~kJ}$ (1800 kcal) and $10251 \mathrm{~kJ}$ (2450 kcal) for men, the mean BMI indicates that the population was obese. Hypertension was most prevalent among AfricanAmerican females (53\%) and lowest among White females (36\%). The prevalence of hypertension among AfricanAmerican and White men was about $44 \%$.

\section{Dietary collection method}

The USDA AMPM dietary recall survey software was used to collect both dietary recalls ${ }^{(22)}$. The survey was supplemented by measurement aids such as measuring cups, spoons, a ruler and an illustrated Food Model Booklet to assist participants in estimating accurate quantities of foods and beverages consumed. Trained interviewers administered both $24 \mathrm{~h}$ dietary recalls. The USDA five-step multiple-pass method has been validated as an accurate methodology for assessing intakes of macronutrients and energy in obese and non-obese men and women ${ }^{(22,23)}$. Eating occasions were self-reported and included breakfast, brunch, lunch, dinner, supper, snack, beverage and extended consumption. For the present study the eating occasions are referred to as 'mealtimes'. The source of where the food was obtained was also collected. Foods reported as consumed for the following three eating occasions: as a snack, beverage or extended consumption, were excluded from the present analysis.

The dietary recalls were coded using the FNDDS version 3.0 in the Survey Net coding software ${ }^{(24)}$. The nine major food groups in the FNDDS were expanded to fiftyeight groups for the present study to separate groups by their fat, sugar and $\mathrm{Na}$ contents, as well as by the degree of processing such as refined $v$. whole grains. Combination codes assigned initially by AMPM were reviewed in Survey Net, providing the coder the ability to change, remove or add new codes to ensure that foods eaten together were correctly linked $^{(4,25)}$. Combinations were defined using two separate variables: (i) a combination food number, which distinguishes foods as eaten in combination; and (ii) combination food type. There were fourteen combination types defined by the USDA Food Coding Scheme (beverage, cereal, bread/baked product, salad, sandwich, soup, frozen meal, ice cream, dried beans/vegetable, fruit, tortilla, meat/poultry/fish, lunchables and chips), excluding a category of ' 99 - other food mixtures'. For the present study, the researchers created five additional combination types based on foods found in the '99' category (pasta dishes, rice dishes, Asian dishes, pizza and dairy). A total of nineteen unique combination types were used for analysis. Examples of combinations include beverages with additions such as added sugar and dairy products, sandwiches, salads and bread/baked goods with additions such as jelly to bread.

\section{Statistical analyses}

Duplicate analysis methods were performed on two separate data sets. Data Set-Original consisted of all foods where each food item retained its original respective USDA food code. This data set includes foods that are reported consumed as individual items and selected composite (i.e. already coded as combinations) foods. Examples of composite foods include spaghetti with meat sauce and brand name fast-food items (McDonald's cheeseburger).

The final data set (Data Set-Revised) was derived exclusively from the Data Set-Original. First, foods eaten in combination (i.e. containing combination codes) were isolated from those without combination codes. The data 
set of foods with combination codes was then modified to reflect their concurrent consumption as a single food item. Specifically, foods consumed simultaneously (as a distinct food item) were aggregated into one food record and given a new individual food code. The final step appended the newly aggregated foods with the remaining noncombination foods into the final revised data set (Fig. 2).

The frequency of reported intake over both days of dietary recalls was calculated and categorized into one of fifty-eight USDA food groups. Since foods eaten simultaneously that were assigned combination codes represent multiple food groups, the main food component was used to define the appropriate food group (Data Set-Revised). For example, white bread toast with butter and jam was associated with refined breads. Next, the frequency of reported intake was calculated for all foods consumed in Data Set-Original along with foods consumed in Data SetRevised. Initially, there were 57543 lines of food codes. Of this count, 26231 lines (46\% of the data set) represented foods assigned combination codes for linkage. When the combinations were aggregated, the number of lines was reduced to 8644 , representing $21.6 \%$ of the records in Data Set-Revised (Fig. 2).
For the present study, frequencies of consumption of food groups by the HANDLS population for three mealtimes, breakfast, lunch and dinner, were generated. Since the reported consumption of foods at brunch and supper was small, $1 \%$ and $2 \%$ of the total number of lines of food codes, respectively, items consumed for brunch were included in breakfast and foods reported eaten as supper were included with dinner. These three main meals accounted for $72.3 \%$ of the non-combined original dietary data. The most frequently consumed foods from selected food groups were determined.

For lack of a clear cut-point in the descriptive frequency analyses, the top twelve food groups in each of the three mealtimes, namely breakfast, lunch and dinner, were selected as a representative majority of the foods eaten (Table 3). These top twelve food groups represented a minimum of $70 \%$ of the top foods eaten per occasion (breakfast $81 \%$, lunch $74 \%$, dinner $72 \%$ ). Together, the food groups in Table 3 represented $86 \%$ (40 336/46 765) of all foods reportedly eaten. It should be noted that the total percentage contributed by the top twelve food groups for each mealtime was always greater when using Data SetRevised compared with Data Set-Original (Table 3).

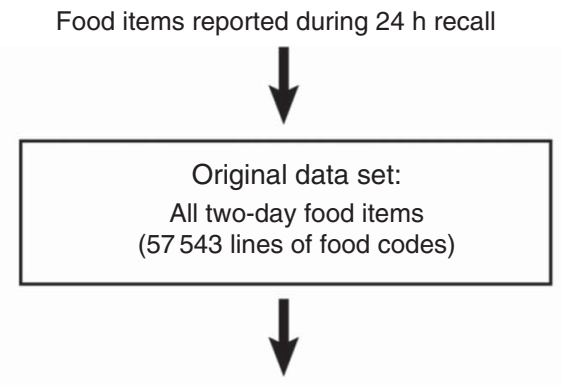

Food item lines partitioned into two data sets: foods with combination codes that were not initially aggregated; and the remainder coded foods

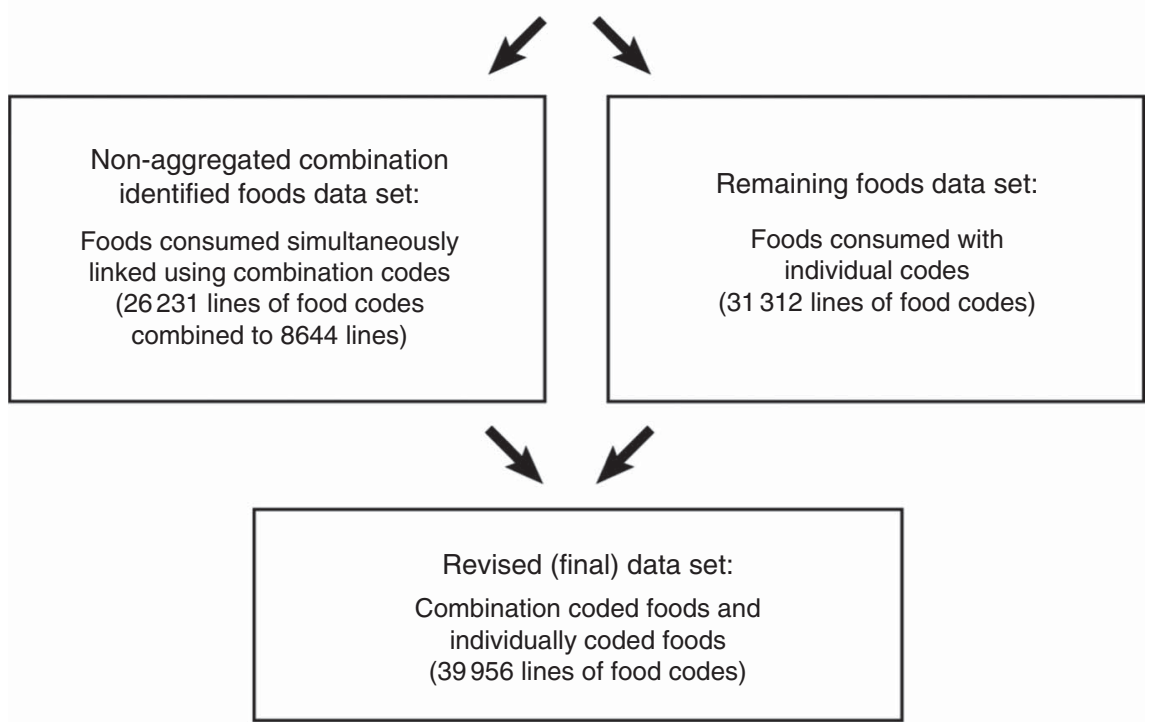

Fig. 2 Construction of food data sets for the present study 
Table 3 Typical meals of an urban population ( $n$ 2177) defined by frequency of reported use: a comparison of the original data set (coding dietary recalls without the use of combination codes) and the revised data set (coding dietary recalls using combination codes), Healthy Aging in Neighborhoods of Diversity across the Life Span (HANDLS) study

Data Set-Original

(all coded foods entered analysis as individual items)

\begin{tabular}{|c|c|c|c|}
\hline \multicolumn{2}{|c|}{ (all coded foods entered analysis as individual items) } & \multicolumn{2}{|c|}{ (coded foods identified as combinations entered analysis as aggregates) } \\
\hline Food group & $\%$ & Food group & $\%$ \\
\hline \multicolumn{4}{|l|}{ Breakfast } \\
\hline Coffee and tea, unsweetened & $10 \cdot 79$ & Eggs and egg dishest† & $12 \cdot 76$ \\
\hline Refined yeast and quick breads & 9.93 & Beverages with sugarłł & $12 \cdot 65$ \\
\hline Sugar & 8.68 & Refined yeast and quick breads & $11 \cdot 25$ \\
\hline Eggs and egg dishes* & $8 \cdot 19$ & Sausage, bacon, luncheon meats & 8.53 \\
\hline Sausage, bacon, luncheon meats & 7.62 & Ready to eat cereals & $7 \cdot 34$ \\
\hline Regular dairy products & 4.87 & Coffee and tea, unsweetened & $6 \cdot 89$ \\
\hline Ready to eat cereals & 4.71 & Citrus fruit & $5 \cdot 38$ \\
\hline Reduced fat dairy products & 3.78 & Diet drinks & $5 \cdot 12$ \\
\hline Citrus fruit & 3.57 & Fruit & 4.63 \\
\hline Beverages with sugart & 3.57 & Cakes, doughnuts, pastries & 3.60 \\
\hline Fruit & 3.46 & Sandwich§§ & 3.26 \\
\hline Regular milk & 2.98 & Cooked cereals & $3 \cdot 17$ \\
\hline TOTAL & $72 \cdot 15$ & TOTAL & $80 \cdot 98$ \\
\hline \multicolumn{4}{|l|}{ Lunch } \\
\hline Other vegetables $\ddagger$ & 11.09 & Sandwich§§ & $15 \cdot 91$ \\
\hline Refined yeast and quick breads & 9.99 & 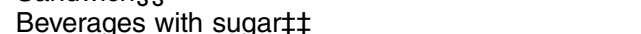 & $15 \cdot 61$ \\
\hline Beverages with sugart & $9 \cdot 14$ & Diet drinks & $6 \cdot 32$ \\
\hline Sausage, bacon, luncheon meats & 6.07 & Starchy vegetables \|\|$\|$ & 5.53 \\
\hline Animal fats & 3.95 & Salty snacks & 5.42 \\
\hline Condiments & 3.89 & Other vegetables & 4.76 \\
\hline Meat dishes§ & 3.72 & Meat dishes & 4.01 \\
\hline Diet drinks & 3.71 & Fruit, raw, canned, or frozen and $100 \%$ fruit juices & 3.59 \\
\hline Starchy vegetables\|l & 3.60 & Chicken and turkeyqศ & 3.54 \\
\hline Salty snacks & 3.42 & Pasta and pasta dishes & 3.32 \\
\hline Chicken and turkey & 2.98 & Coffee and tea, unsweetened & $3 \cdot 13$ \\
\hline Coffee and tea, unsweetened & 2.83 & Refined yeast and quick breads & $3 \cdot 11$ \\
\hline TOTAL & 64.39 & TOTAL & 74.25 \\
\hline \multicolumn{4}{|l|}{ Dinner } \\
\hline Other vegetables $\ddagger$ & $12 \cdot 00$ & Beverages with sugarłł & $13 \cdot 28$ \\
\hline Beverages with sugart & 9.02 & Starchy vegetables \|\|$\|$ & $9 \cdot 77$ \\
\hline Starchy vegetablesil & $7 \cdot 30$ & Other vegetables & $7 \cdot 50$ \\
\hline Refined yeast and quick breads & $7 \cdot 27$ & Sandwich§§ & $7 \cdot 18$ \\
\hline Chicken and turkey & 4.70 & Chicken and turkey & 5.81 \\
\hline Meat dishes§ & 4.37 & Meat dishes & 5.49 \\
\hline Animal fats & 3.73 & Pasta and pasta dishes & $5 \cdot 24$ \\
\hline Pasta and pasta dishes & 3.69 & Refined yeast and quick breads & 4.95 \\
\hline Condiments & 3.05 & Diet drinks & 4.03 \\
\hline Coffee and tea, unsweetened & 2.83 & Coffee and tea, unsweetened & 3.01 \\
\hline Sausage, bacon, luncheon meats & $2 \cdot 82$ & Dark green vegetables & $2 \cdot 81$ \\
\hline Red meats** & 2.65 & Rice and rice dishes & 2.78 \\
\hline TOTAL & 63.43 & TOTAL & 71.85 \\
\hline
\end{tabular}

${ }^{\star}$ Egg and egg dishes include cooked eggs, omelettes and egg sandwiches pre-prepared.

†Beverages with sugar include soft drinks, pre-sweetened tea, coffee and water beverages, fruit drinks and sports drinks.

$\ddagger$ Other vegetables include vegetables that are not orange, dark green or starchy. Vegetable could be consumed raw or cooked with or without fat.

§Meat dishes include frozen and prepared dishes, dumplings and egg rolls with red meats, chicken or fish, and Hispanic dishes with meat.

\|Starchy vegetables include potatoes, corn, peas, plantain and beans prepared with or without fat.

ๆChicken and turkey include poultry items (parts or processed like nuggets) prepared with fat and eaten with skin.

${ }^{* *}$ Red meats include meats with greater than $10 \mathrm{~g}$ total fat, $4.5 \mathrm{~g}$ saturated fat and $95 \mathrm{mg}$ cholesterol per $\sim 100 \mathrm{~g}(3.5 \mathrm{oz})$ serving and meats prepared with fat. ††Egg and egg dishes include cooked eggs, omelettes, and egg sandwiches pre-prepared and prepared using individual ingredients.

$\ddagger \ddagger B$ everages with sugar include soft drinks, pre-sweetened tea, coffee and water beverages, fruit drinks, sports drinks and beverages to which sugar was added by an individual.

$\S \S$ Sandwiches include all types with the exception of egg as the main ingredient. The major ingredient could be beef, pork, chicken, turkey, fish, bacon, sausage, luncheon meats, hot dogs or vegetables.

IIIIStarchy vegetables include potatoes, corn, peas and beans prepared with or without fat, and these items with added condiments like ketchup (catsup) and fats. ITTChicken and turkey include poultry items (parts or processed like nuggets) prepared with fat and eaten with skin along with an added condiment, gravy or sauce.

To further assess the impact of coding method, the rank order of food groups by race was determined. For this analysis, data for the three mealtimes were merged together, resulting in a representation of intake on $24 \mathrm{~h}$ recall days (Table 4). Statistical analyses were performed with the SAS statistical software package version $9 \cdot 2$.

\section{Results}

To our knowledge, the present publication is the first one that compares food intakes with and without using the combination codes that are in WWEIA, NHANES as well as the HANDLS study. As shown in Table 3, when foods 
Table 4 Foods consumed frequently by urban African-American and White adults: a comparison of rank-ordered food groups by coding method within and between races* Healthy Aging in Neighborhoods of Diversity across the Life Span (HANDLS) study

Data Set-Original

(all coded foods entered analysis as individual items)

\begin{tabular}{|c|c|c|c|c|c|c|c|}
\hline \multicolumn{4}{|c|}{ (all coded foods entered analysis as individual items) } & \multicolumn{4}{|c|}{ (coded foods identified as combinations entered analysis as aggregates) } \\
\hline \multicolumn{2}{|l|}{ African Americans } & \multicolumn{2}{|l|}{ Whites } & \multicolumn{2}{|l|}{ African Americans } & \multicolumn{2}{|l|}{ Whites } \\
\hline Food group & $\%$ & Food group & $\%$ & Food group & $\%$ & Food group & $\%$ \\
\hline Refined yeast and quick breads & 8.93 & Other vegetables $\ddagger$ & 9.90 & Beverages with sugar** & $15 \cdot 21$ & Beverages with sugar** & 11.62 \\
\hline Beverages with sugart & 8.68 & Refined yeast and quick breads & 8.79 & Sandwich†† & 7.68 & Sandwicht† & 9.11 \\
\hline Other vegetables $\ddagger$ & 7.68 & Coffee and tea, unsweetened & $6 \cdot 20$ & Starchy vegetablesł‡ & 6.63 & Diet drinks & 6.79 \\
\hline Sausage, bacon, luncheon meats & 6.00 & Beverages with sugar† & 5.87 & Refined yeast and quick breads & $6 \cdot 31$ & Starchy vegetablesł‡ & 6.35 \\
\hline Starchy vegetables§ & 4.69 & Starchy vegetables§ & 4.25 & Eggs and egg dishes§§ & 5.02 & Refined yeast and quick breads & 5.96 \\
\hline Coffee and tea, unsweetened & 4.38 & Sausage, bacon, luncheon meats & $4 \cdot 17$ & Chicken and turkeyllII & 4.96 & Coffee and tea, unsweetened & $5 \cdot 60$ \\
\hline Sugar & 3.99 & Diet drinks & 3.44 & Sausage, bacon, luncheon meats & 4.31 & Other vegetables & 5.57 \\
\hline Chicken and turkeyll & 3.92 & Animal fats & 3.34 & Other vegetables & $4 \cdot 19$ & Meat dishes $\uparrow \uparrow$ & 3.89 \\
\hline Eggs and egg dishes $\mathbb{1}$ & 3.56 & Meat dishes & 3.06 & Meat dishes & 3.60 & Eggs and egg dishes§§ & 3.52 \\
\hline Animal fats & 3.35 & Sugar & $2 \cdot 86$ & Diet drinks & 3.57 & Fruit & 3.44 \\
\hline Meat dishes & 3.05 & Fruit & 2.69 & Pasta and pasta dishes & 3.43 & Pasta and pasta dishes & 3.35 \\
\hline Condiments & 2.75 & Condiments & 2.55 & Coffee and tea, unsweetened & 3.09 & Ready to eat cereals & 3.08 \\
\hline Pasta and pasta dishes & 2.29 & Regular dairy products & 2.40 & Fruit & $2 \cdot 88$ & Cakes, donuts, pastries & 2.93 \\
\hline TOTAL & $63 \cdot 27$ & TOTAL & 59.52 & TOTAL & 70.88 & TOTAL & $71 \cdot 21$ \\
\hline
\end{tabular}

*Based on the following three eating occasions: breakfast + brunch, lunch, dinner + supper. Eating occasions identified as snacks, beverages or extended consumption were excluded from the analysis. †Beverages with sugar include soft drinks, pre-sweetened tea, coffee and water beverages, fruit drinks and sports drinks.

fOther vegetables include vegetables that are not orange or dark green or starchy. Vegetable could be consumed raw or cooked with or without fat.

$\S$ Starchy vegetables include potatoes, corn, peas, plantain and beans prepared with or without fat.

"Chicken and turkey include poultry items (parts or processed like nuggets) prepared with fat and eaten with skin.

IEgg and egg dishes include cooked eggs, omelettes and egg sandwiches pre-prepared.

"Beverages with sugar include soft drinks, pre-sweetened tea, coffee and water beverages, fruit drinks, sports drinks and beverages to which sugar was added by an individual.

†TSandwiches include all types with the exception of egg as the main ingredient. The major ingredient could be beef, pork, chicken, turkey, fish, bacon, sausage, luncheon meats, hot dogs or vegetables.

¥\$Starchy vegetables include potatoes, corn, peas and beans prepared with or without fat, and these items with added condiments like ketchup (catsup) and fats.

$\S \S$ Egg and egg dishes include cooked eggs, omelettes, and egg sandwiches pre-prepared and prepared using individual ingredients.

ili Chicken and turkey include poultry items (parts or processed like nuggets) prepared with fat and eaten with skin along with an added condiment, gravy or sauce.

TाMeat dishes include frozen and prepared dishes, dumplings and egg rolls with red meats, chicken or fish, and Hispanic dishes with meat. 
eaten in combination were coded as an aggregate, not only did the percentage contribution of food groups change, but also new food groups appeared. Some food groups such as condiments and sugar disappeared from the list because they were eaten as an addition to foods, namely sandwiches and beverages, respectively.

For breakfast, when the ingredients for self-prepared egg sandwiches were combined and linked to the eggs and eggs dishes food group (Data Set-Revised), the rank order for these items changed from fourth to first place (Table 3). When coffee and tea with sugar were recoded to the beverages with sugar group (Data Set-Revised), the percentage of reported usage of beverages with sugar at breakfast increased from $3.57 \%$ to $12.65 \%$ (Table 3), making it the second highest used group. Of the beverages with sugar group, $48 \%$ of the foods were sweetened coffee, $32 \%$ fruit-flavoured drinks and soda and $20 \%$ were sweetened tea. In addition to the disappearance of sugar from Data Set-Original, three other groups, namely regular dairy products, reduced-fat dairy products and regular milk, disappeared. This disappearance of regular milk and reduced-fat dairy products, which included reduced-fat milks, most likely reflects their use as a combination with ready-to-eat cereals (19\% of dairy products) and in coffee and tea ( $68 \%$ of dairy products). The new groups that appeared when using Data Set-Revised were diet drinks, cakes, doughnuts and pastries, sandwiches and cooked cereals (Table 3).

For lunch, most of the changes in the rank order reflected the aggregation of food items for self-prepared sandwiches (Table 3). From Data Set-Original, other vegetables moved from first to sixth place; refined breads moved from second to twelfth place; and sausage, bacon and luncheon meats, animal fats and condiments disappeared. The reported usage of sandwiches at lunch was $15.91 \%$, making it the top food group in Data Set-Revised (Table 3). Of the sandwich category, sandwiches containing red meats (mainly beef) ranked first $(54 \%)$ followed by sandwiches containing chicken (20\%), fish (9\%) or hot dogs (9\%). Similar to breakfast, beverages with sugar ranked second when foods eaten in combination were aggregated. However, unlike breakfast, fruit-flavoured drinks such as Kool-Aid and sodas were the largest contributor to this group at $77 \%$, followed by sweetened tea (19\%) and then coffee (4\%). The rank order of both starchy vegetables and salty snacks moved upward in Data Set-Revised. Fruit (eighth place) and pasta dishes (tenth place) appeared only in Data Set-Revised (Table 3).

For dinner, the other vegetable category, which excludes dark green, orange and starchy vegetables, moved from first to third place when combination coding was used (Table 3). This change most likely reflected the incorporation of lettuce and tomatoes into sandwiches. There were four food groups, namely animal fats, condiments, sausage, bacon and luncheon meats and red meat, in Data Set-Original that disappeared with the use of combination coding. The rank order of refined breads dropped from fourth place to eighth place. Most likely these changes can be explained by the incorporation of these food items into the sandwich group. The types of sandwiches were similar to lunch - red meatbased (59\%), chicken-based (16\%), fish-based (9\%) and hot dogs (10\%). The new groups that appeared in Data SetRevised were dark green vegetables, diet drinks, and rice and rice dishes.

Further evidence that coding method does impact the results of daily intake dietary analysis was demonstrated by a comparison of the rank-ordered food intake by race. As shown in Table 4, eight of the thirteen food groups in Data Set-Original appeared for both the African-American and White adults examined in the HANDLS study. However, the rank order of these food groups, with the exception of starchy vegetables and condiments, differed by race. When combination codes were used (Data SetRevised), there was no racial difference for the top two food groups. The foods from the beverages with sugar and sandwich groups were the most frequently consumed by both the African-American and White HANDLS study participants (Table 4). There were racial differences in intakes of sausage, bacon and luncheon meat and chicken/turkey groups, which were among the most frequently reported as consumed by African Americans. In comparison, ready-to-eat cereals and cakes, doughnuts and pastries were among the most frequently reported foods consumed by Whites during the $24 \mathrm{~h}$ recall.

\section{Discussion}

Using the USDA AMPM, investigators have the ability to study foods consumed in combination and their nutrient profiles. However, the use of combination codes is rarely cited in publications. The findings of the present research demonstrate differences in rank-ordered food intakes for mealtime and in rank-ordered daily food intakes for race based upon food coding methods used with multiple-day dietary recall data. These changes in rank ordering of food/food groupings can result in a change in the characterization of the population's dietary patterns when attempting to quantify patterns using current epidemiological methods, such as cluster, principal component or factor analyses or reduced rank regression ${ }^{(10)}$.

Despite the amount of time required, as well as the intricate and subjective choices of applying the combination coding to almost half of the total foods reported, this approach better reflects how individual foods were actually consumed. This knowledge permits researchers to have a more comprehensive description of a meal and to allow better comparisons of foods consumed by eating occasion. For instance, the similarities between the lunch and dinner meal for this US population segment became clearly evident with combination coding. The typical lunch or dinner for the HANDLS study participant consisted of a 
sandwich, starchy vegetable and beverages with sugar. The majority of these items had combination codes. The use of combination codes when analysing daily intakes made similarities and differences by race easily identifiable.

Similar to a dietary collection method, the coding method should be based on the practice/research objectives. The choice of coding method is illustrated in the following examples. If the identification of foods as typically consumed is the objective, then using a coding approach that aggregates foods eaten in combination (or simultaneously) would be the recommended method. An interesting finding for this urban population is that sandwiches were consumed at each eating occasion, most frequently at lunch. However, this observation was seen only when the coding method involved aggregating the items eaten in combination. If the objective of the research is to examine the quantity (millilitres) of beverages consumed, coding of the individual items would be more appropriate. On the other hand, if researchers want to compare the energy contribution of beverages to total dietary energy among population groups, then the coding must include combined items. Otherwise, energy would be underestimated by the additions (e.g. sugar, milk, honey, etc.) to items such as coffee and tea.

Even though time is required for a professional to code and map each combination to the appropriate food group, and for a statistician to program the analyses, one strength of coding foods as typically consumed is that it provides a better representation of eating behaviour. In addition, it allows for the formulation of policies and interventions targeted to these food preferences. It is widely recognized that the US population needs to consume more vegetables and fruits. If the analysis reveals that the study population does not consume salad on a regular basis but sandwiches are frequently consumed, nutritionists can recommend ways to incorporate vegetables into sandwiches rather than trying to promote salads. Cluster, principal component and other dietary pattern analyses using the combination codes in a database can reveal more recognizable and interpretable patterns. These patterns may then be associated with impacting health and chronic disease ${ }^{(10)}$. No matter what approach to coding a researcher selects it is important for investigators to describe the procedure used to code the data.

Knowing the source of food as it relates to food combinations helps to further our understanding of food availability, food choice and the types of foods people self-prepare. The majority of foods consumed as individual or combined items, except for sandwiches, were obtained from the store, with a range of $62-92 \%$. Fastfood restaurants were the primary source of ready-to-eat sandwiches ( $79 \%$ of sandwiches reported). Thus the participants in the HANDLS study were doing some food preparation, which would support implementation of culinary-based interventions. Knowledge of how foods were prepared prior to consumption based on combination coding can aid in defining more healthful food choices that may improve diet quality.

Lastly, the present study provides information on a lowincome urban population of African-American and White adults which are an understudied group. Consistent with the findings of James on a convenience sample of AfricanAmerican adults ${ }^{(26)}$, food intakes of African-American HANDLS study participants were not consistent with current Dietary Guidelines for Americans ${ }^{(27)}$. Oftentimes dietary guidance is targeted to individual foods and/or nutrients rather than meal choices: for instance, replace protein foods that are higher in solid fats with choices that are lower in solid fats and calories and/or are sources of oils ${ }^{(27)}$. Perhaps the majority of people do not perceive food as presented in this type of guidance but think of food as consumed - a double burger with cheese rather than a grilled chicken sandwich with lettuce and tomato. Using combination codes may provide educators better insight to dietary practices which can be valuable when developing targeted nutrition-related messages.

The present results describe a population that resided in Baltimore, MD. However, independent demographic analyses produced findings supporting that this population was representative of urban populations from US cities with similar population densities and racial distribution. These cities include Atlanta, GA; Bridgeport, CT; Bridgeton, NJ, Buffalo, NY; Camden, NJ; Carson, CA; Chicago, IL; Cleveland, OH; Detroit, MI; Harrisburg, PA; Hartford, CT; Oakland, CA; Springfield, MS; and Trenton, NJ (J Lepkowski, unpublished results).

Sometimes the use of the combination codes approach may not be the preferred coding method. If the objective of the research is to compare intakes with food group recommendations, a coding method that aggregates combinations would result in an underestimation of selected food groups. For example, if a researcher was interested in servings of dairy, dairy food group servings would be underestimated if the cheeses added to pasta were coded as an aggregate and assigned to the pasta dish food group. However, using the USDA AMPM with FNDDS, retention of the original data set that consists of each individually coded food item mentioned in a recall and the disaggregation of coded mixed dishes and brandname items can be used for such analyses.

\section{Conclusion}

In conclusion, the method of coding dietary data does influence results and the coding approach should be consistent within a data set. Mixtures (such as egg dishes or sandwiches) should all be coded as individual ingredients or as composites. The advantage of using a coding approach utilizing food combinations is that it provides a better picture of how people are eating. These findings may be valuable to health professionals enabling them to formulate more 
focused, user-friendly public health messages for vulnerable populations on how to modify their diet to make it more healthful. Accurate knowledge of how people choose and combine the foods they consume may provide better insights for nutrition researchers and policy makers on the relationships between diet and health.

\section{Acknowledgements}

Financial support: This work was supported by the Intramural Research Program, National Institute on Aging, National Institutes of Health (NIH). NIH had a role in the design, analysis or writing of this article. Conflict of interest: None. Authorship: M.A.M., M.F.K., D.A., A.B.Z. and M.K.E. designed and conducted the research. M.F.K. and D.A. coded dietary data and M.A.M. analysed the data. M.F.K., M.A.M., D.A., A.B.Z. and M.K.E. wrote the manuscript. M.A.M. and M.F.K. had primary responsibility for the final content. All authors read and approved the final manuscript. Etbics of buman subject participation: All procedures were approved by the Institutional Review Boards at both MedStar Health Research Institute and the University of Delaware.

\section{References}

1. Park SY, Murphy SP, Wilkens LR et al. (2005) Dietary patterns using the Food Guide Pyramid groups associated with sociodemographic and lifestyle factors: the Multicohort Study. J Nutr 135, 843-849.

2. Tucker KL (2010) Dietary patterns, approaches, and multicultural perspective. Appl Physiol Nutr Metab 35, 211-218.

3. Fulgoni VL III \& Quann EE (2012) National trends in beverage consumption in children from birth to 5 years: analysis of NHANES across three decades. Nutr J 11, e92.

4. Raper N, Perloff B, Ingwersen L et al. (2004) An overview of USDA's Dietary Intake Data System. J Food Compost Anal 17, 545-555.

5. Centers for Disease Control and Prevention (2014) NHANES 2009-2010 Dietary Data. http://wwwn.cdc.gov/nchs/ nhanes/search/datapage.aspx?Component=Dietary\&Cycle BeginYear=2009 (accessed October 2014).

6. National Institute on Aging (2010) HANDLS (Healthy Aging in Neighborhoods of Diversity across the Life Span Homepage). http://www.handls.nih.gov (accessed October 2014).

7. Kerver JM, Yang EJ, Bianchi L et al. (2003) Dietary patterns associated with risk factors for cardiovascular disease in healthy US adults. Am J Clin Nutr 78, 1103-1110.

8. Austin GL, Ogden LG \& Hill JO (2011) Trends in carbohydrate, fat, and protein intakes and association with energy intake in normal-weight, overweight, and obese individuals: 1971-2006. Am J Clin Nutr 93, 836-843.

9. Fanelli Kuczmarski M, Mason MA, Allegro D et al. (2013) Diet quality is inversely associated with C-reactive protein levels in urban, low-income African-American and White adults. J Acad Nutr Diet 113, 1620-1631.

10. Fanelli Kuczmarski M, Mason MA, Beydoun M et al. (2013) Dietary patterns and sarcopenia in an urban African
American and White population in the United States. J Nutr Geriatr Geronotol 32, 291-316.

11. Han E \& Powell LM (2013) Consumption patterns of sugarsweetened beverages in the United States. J Acad Nutr Diet 113, 43-53.

12. Kit BK, Fakhouri THI, Park S et al. (2013) Trends in sugarsweetened beverage consumption among youth and adults in the United States: 1999-2010. Am J Clin Nutr 98, 180-188.

13. Paeratakul S, Ferdinand DP, Champagne CM et al. (2003) Fast-food consumption among US adults and children: dietary and nutrient intake profile. J Am Diet Assoc 103, $1332-1338$.

14. Mullie P, Vansant G, Hulens M et al. (2010) Dietary patterns and socioeconomic position. Eur J Clin Nutr 64, 231-238.

15. Martikainen P, Brunner E \& Marmot M (2003) Socioeconomic differences in dietary patterns among middleaged men and women. Soc Sci Med 56, 1397-1410.

16. Kumanyika SK \& Krebs-Smith SM (2000) Preventive nutrition issues in ethnic and socioeconomic groups in the United States. In Preventive Nutrition. vol. II: Primary and Secondary Prevention, pp. 325-356 [A Bendich and RJ Deckelbaum, editors]. Totowa, NJ: Humana Press Inc.

17. Taylor CA, Gilmore AC \& Keim KS (2005) Impact of core and secondary foods on nutritional composition of diets in Native American women. J Am Diet Assoc 105, 413-419.

18. Sirot V, Volatier JL, Calamassi-Tran G et al. (2006) Core food of the French food supply: second Total Diet Study. Food Addit Contam 26, 623-639.

19. Evans MK, Lepkowski JM, Powell NR et al. (2010) Healthy Aging in Neighborhoods of Diversity across the Life Span (HANDLS): overcoming barriers to implementing a longitudinal, epidemiologic, urban study of health, race, and socioeconomic status. Ethn Dis 20, 267-275.

20. Evans MK \& Zonderman AB (2004) Healthy Aging in Neighborhoods of Diversity across the Life Span. HANDLS Research Protocol. Version No: 3. http://handls.nih.gov/ pdf/HANDLS-2Protocol-2004-10-08-Protocol.pdf (accessed October 2014).

21. US Department of Health and Human Services (2003) The 2003 HHS Poverty Guidelines. http://aspe.hhs.gov/poverty/ 03poverty.htm (accessed October 2014).

22. Moshfegh AJ, Rhodes DG, Baer DJ et al. (2008) The US Department of Agriculture automated multiple-pass method reduces bias in the collection of energy intakes. Am J Clin Nutr 88, 324-332.

23. Conway JM, Ingwerson LA \& Moshfegh AJ (2004) Accuracy of dietary recall using the USDA five-step multiple-pass method in men: an observational validation study. J Am Diet Assoc 104, 595-603.

24. Agricultural Research Service, Food Surveys Research Group (2014) USDA Food and Nutrient Database for Dietary Studies, 3.0. http://www.ars.usda.gov/Services/docs.htm? docid=12089 (accessed October 2014).

25. Centers for Disease Control and Prevention. (2013) NHANES dietary web tutorial. Resources for dietary data analysis. http://www.cdc.gov/nchs/tutorials/Dietary/Survey Orientation/ResourceDietaryAnalysis/intro.htm (accessed October 2014).

26. James DCS (2009) Cluster analysis defines distinct dietary patterns for African-American men and women. $J$ Am Diet Assoc 109, 255-262.

27. US Department of Agriculture \& US Department of Health and Human Services (2013) Dietary Guidelines for Americans 2010. http://www.cnpp.usda.gov/dgas2010policydocument.htm (accessed October 2014). 